\title{
Local level spatiotemporal dynamics of the COVID-19 transmission in the Greater Seoul Area: a view from a Bayesian perspective
}

\author{
Authors and affiliations : \\ Youngbin Lym PhD (First Author) \\ Research Institute of Natural Sciences, Chungnam National University \\ 99 Daehak-ro, Yuseong-gu, Daejeon 34134, Republic of Korea \\ Tel: 82-10-4693-4632 Email: youngbin.lym@gmail.com \\ Hyobin Lym (Co-author) \\ Center for Agricultural Outlook Sejong Office, Korea Rural Economic Institute \\ 4th floor conference room, 150, Osongsaengsaeng 3-ro, Osong-eup, Heungdeok-gu, Cheongju-si, \\ Chungcheongbuk-do, 28165, Republic of Korea \\ Tel: 82-43-238-3068 Email: hyobin@krei.re.kr \\ Ki-Jung Kim PhD (Corresponding author) \\ Assistant Professor \\ Department of Smart Car Engineering, Doowon Technical University \\ 159 Jurawu-gil, Paju-eup, Paju-si, Gyeonggi-do, 10838, Republic of Korea \\ Tel:82-31-935-7323 Email:kimkj@doowon.ac.kr \\ Corresponding Author : \\ Ki-Jung Kim PhD \\ Assistant Professor \\ Department of Smart Car Engineering, Doowon Technical University \\ 159 Jurawu-gil, Paju-eup, Paju-si, Gyeonggi-do, 10838, Republic of Korea \\ Tel:82-31-935-7323 Email: kimkj@doowon.ac.kr
}

\section{Ethics statement}

Not applicable.

\section{Conflict of interest}

The authors declare that they have no known competing financial interests or personal relationships that could have appeared to influence the work reported in this paper.

\section{Funding}

Youngbin Lym (Y.L) was supported by Basic Science Research Program through the National Research Foundation of Korea funded by the Ministry of Education (NRF-

2021R1A6A3A01087232).

\section{Acknowledgement}

The authors would like to thank Statistical Geographic Information Service (SGIS) for providing the geospatial data. Views presented in this study do not represent any organizations and authors are responsible for any errors or mistakes. 


\section{Author contributions}

Conceptualization: YL, K-JK. Data curation: YL, HL, K-JK. Formal analysis: YL, HL. Methodology: YL. Software: YL. Writing — Original draft: YL, HL, K-JK. Writing — Review \& editing: YL, K-JK.

Visualization: YL, K-J.K. Investigation: YL, K-J.K. Validation: YL, K-J.K. Funding acquisition: YL. Project administration: K-J.K.

\section{ORCID}

Youngbin Lym: https://orcid.org/0000-0001-5903-8618

Hyobin Lym: https://orcid.org/0000-0001-8519-8826

Ki-Jung Kim: https://orcid.org/0000-0001-6237-6896 


\title{
Local level spatiotemporal dynamics of the COVID-19 transmission in the Greater Seoul Area: a view from a Bayesian perspective
}

\begin{abstract}
Objectives: The purpose of this research is to enhance the understanding of the local-level spatiotemporal dynamics of COVID-19 diffusion in the Greater Seoul Area (GSA), South Korea, after its initial outbreak in January 2020.

Methods: Using the weekly aggregates of COVID-19 cases of 77 municipalities in the GSA, we attempt to examine the relative risks of COVID-19 infections across local districts over the fiftyconsecutive weeks in 2020. To this end, we employ a spatiotemporal generalized linear mixed model under the hierarchical Bayesian framework. This allows us to empirically examine the random effects of spatial alignments, temporal autocorrelation, and spatiotemporal interaction along with the fixed effects. Specifically, we exploit the intrinsic conditional autoregressive and the weakly informative penalized complexity priors for hyperparameters of the random effects.
\end{abstract}

Results: Our study reveals that spatiotemporal interaction dominates the overall variability of random influences, followed by spatial correlation, whereas the temporal correlation appears to be small. Considering these findings, we present the dynamic evolution of COVID-19 across local municipalities in the GSA as well as regions of an elevated risk for further policy intervention.

Conclusions: The outcomes of this study can contribute to advancing our understanding of the locallevel COVID-19 spread dynamics within densely populated regions in South Korea throughout 2020 from a different perspective, leading to devising regional safety planning against infectious diseases.

Keywords: COVID-19, Hierarchical Bayesian, Spatiotemporal transmission dynamics, Local municipalities, Relative risks 


\section{INTRODUCTION}

As of December 31, 2020, more than 82 million COVID-19 cases were confirmed worldwide, with more than 1.8 million deaths attributed to the infectious disease [1]. According to the Korea Disease Control and Prevention Agency (KDCA), there have been a total of 60,740 confirmed cases (117 per 100,000 population) of COVID-19 reported with 900 deaths (1.58 per 100,000 population) in 2020 in South Korea [2]. As such, the reason that COVID-19 shows rapid spread around the world is due to the characteristics of respiratory infectious diseases, since they are easily spread through direct or indirect contact with an infected person or through droplets from the infected [3]. In addition, some studies point out that the spread of COVID-19 is caused by the growth in exchanges among regions following the development of transportation means and an increase in the frequency of contact due to high population density [4-5].

In 2020, there has been three heterogeneous waves of the COVID-19 pandemic, including the first in February-March, the second in August-September, and the third in mid-November in South Korea. In response to each wave, the South Korean government introduced and implemented several countermeasures against the disease (e.g., social distancing, mandatory mask wearing, and tracing and tracking of the confirmed patients) to suppress its spread without having a nationwide lockdown. We observed that throughout 2020, the pattern of COVID-19 outbreaks in South Korea has revealed unique features which are assumed to be affected by the government's response to the virus. We also acknowledge that each country has differently responded to COVID-19 so that the outcomes would vary. This leads us to investigate how the infectious virus spreads and evolves across the regions over time.

Numerous attempts have been made to unveil the association between COVID-19 spread/evolution and contributing factors including built environment, environmental conditions, air pollutions, and spatiotemporal dynamics. For instance, Briz-Redón and Serrano-Aroca [6] explored the early evolution of COVID-19 in Spain, considering the effects of temperature, space, and time. Briz-Redón [7] further examined the impact of modeling choices on the outcomes by comparing an array of spatiotemporal model specifications of 42 regions for 182 days in Catalonia, Spain. Kang et 
al. [8] explored the spatial dynamics of the COVID-19 outbreak in China at an early stage using Moran's I, which revealed spatial association among different regions. Valente and Laurini [9] estimated the spatial spreads of mortality by COVID-19 on a global scale during the late February 2020 to middle February 2021, taking the six continents into consideration. When it comes to COVID-19 cases relative to South Korea, Kim and Castro (2020) [10] implemented a retrospective space-time scan statistic to identify 12 spatiotemporal clusters for early period of the pandemic. Recently, Lee, Park, and Lee [11] attempted to unveil the spatiotemporal evolution and diffusion pattern of COVID-19 infections in three waves in South Korea via ArcGIS and network analyses. The outcomes revealed the disparities among three waves of the pandemic in terms of transmission scales, speed, and directions. From a different perspective, Shim et al. [12] showed the spatial variability in the reproduction number and doubling time of infections in South Korea utilizing daily confirmed cases obtained from January to July 2020. Lee, Hwang, and Song et al. [13] examined the daily reproduction number as well as fatality pattern, and then showed the spread pattern based upon a logistic growth model for the initial period of the pandemic. Lee, Kwon, and Lee [14] studied the effect of four control measures - school closure, quarantine, isolation, and social distancing — on the transmission of COVID-19 via an extended age-structured SEIR model.

Although previous efforts have shown their relevance in COVID-19 transmission across space over time, detailed local-level dynamics of the diffusion (i.e., spreads of diseases within a metropolitan region) are yet to be fully explored in South Korea. Hence, motivated by these limitations of previous research endeavors, this study attempts to scrutinize the spatiotemporal evolution of the COVID-19 pandemic at the local municipality level in the Greater Seoul Area (GSA, i.e., the capital region of South Korea) for fifty consecutive weeks of 2020. To elucidate the relative risks of COVID-19 infection, we exploit a full hierarchical Bayesian approach under the Generalized Linear Mixed Modeling (GLMM) framework, which is flexible enough to account for the influences of unobserved heterogeneity originating from spatial alignments as well as temporal dependency. To this end, we argue that a systemic breakdown of latent effects provides us enhanced understanding of COVID-19 evolution 
within the GSA from a different perspective, leading to evidence-based decision making for regional safety against infectious diseases.

\section{MATERIALS AND METHODS}

\section{Background}

Prior to delving into data in detail, we would like to discuss various efforts of Korean public health authorities to keep the virus under control. As shown in Figure 1, South Korea has experienced three heterogeneous waves of COVID-19 (as of Dec.31. 2020) since its outbreak in January 2020.

The first wave started in Daegu, originating from a single source, the religious group "Shincheonji," and at that time, the number of cumulative confirmed cases reached 5,213 [15]. After the first wave, the South Korean government announced guidance for social distancing (SD) to prevent the local spread of COVID-19 by limiting contact among the public [16]. In accordance with the trajectory of the outbreak, SD was selectively adopted as "Social Distancing," "Enhanced Social Distancing," and "Distancing in Daily Life" [17]. Specifically, the government advised to avoid gatherings as well as group activities, and to maintain safe distancing from February 29 to March 21 (SD period). When sporadic infections occurred from March 22 to April 19 (enhanced SD period), people were advised to stay at home, while facilities related to religion, indoor sports, and entertainment were closed [17]. In early May, there were a series of small group infections in the GSA (e.g., Itaewon Club and Coupang Logistics Center) [12], but this was not classified as a pandemic. At the end of June, the South Korean government introduced a systematized three-level SD scheme corresponding to the severity of the outbreak (e.g., the number of daily cases for two weeks). This provided clear-cut public messaging and matching countermeasures [17].

\section{[Figure 1. Here]}

The second wave of the pandemic was attributed to two origins: the Sarang Jeil Church (1,170 cases) and a political rally on August 15 (650 cases). Unlike the first wave, the virus spread outside its initial sources, leading to a series of other small-cluster infections in related facilities and communities. As the spread intensified, the South Korean government upgraded the "SD" stage to 
Level 2 and even applied "enhanced" SD measure level 2 (temporarily), which imposed restrictions on the business operation of restaurants and cafes. As a result, the number of confirmed cases fell below 100 in October, and the government lowered SD to Level 1 (distancing of daily life). The Korea Disease Control and Prevention Agency (KDCA), later promoted by the Korea Centers for Disease Control and Prevention (KCDC), announced a more finely tuned set of SD guidelines on November 7 that better reflected public health conditions on the ground [18]. The three-level SD chart has been extended to a new five-tier SD scheme (i.e., Levels 1, 1.5, 2, 2.5, and 3). Each level was determined based on the average number of daily domestic infections over one week.

Meanwhile, the third wave started in the middle of November, heading into the winter season in which the virus is more active. This has posed greater challenges to control the pandemic because of a series of sporadic infections across local communities, making it harder to trace their transmission routes as compared to previous ones. Moreover, amidst the third wave, the average number of daily confirmed cases reached 1,000 in December 2020.

\section{Data description of the study region}

There were about $59.7 \%$ of the total cumulative cases occurred in the GSA, followed by $36.3 \%$ in non-metropolitan areas and $4.0 \%$ in quarantine stages as of December 2020 in South Korea. Daegu, the epicenter of the first wave, had the highest incidence rate of COVID-19 (320.17 per 100,000), followed by Seoul (195.24), and Gyeonggyeong-do (109.06) [2]. As previously mentioned, the first wave provoked by a church in Daegu did not spill over so that it was suppressed shortly, whereas the spread of COVID-19 for the following waves showed different patterns within the GSA, challenging its control. This motivates us to explore the dynamic evolution of COVID-19 in the capital regions rather than other places with a specific focus of local-level transmission over time.

In that regard, 77 municipalities (i.e., si/gun/gu) within the GSA including Seoul, Gyeonggi province, and Incheon city, are selected as the spatial dimension of this study. For each municipality (district), we initially collected daily confirmed cases of COVID-19 from January 24 (Week 4) to December 31 (Week 53), 2020. We then excluded cases of inbound travelers from foreign countries due to the limitation of the collected data in earlier periods. To improve the validity and accuracy of 
our dataset, we cross-checked it with the confirmed cases of each municipality and updated the data by readjusting reporting-delays or errors. This ensured us to obtain detailed daily time series of COVID-19 data. A weekly aggregate of the outbreak per municipality was utilized for a subsequent investigation. Furthermore, we acquired geospatial data from OpenMarket at the National Spatial Data Infrastructure Portal [19] in order to relate those weekly aggregates and sociodemographic features to spatial data, which were in preparation for the formal statistical assessment. Neighbors of administrative units (i.e., municipalities) were defined when they shared a common border or boundary.

\section{Methodology}

This study adopts a spatiotemporal generalized linear mixed model under a hierarchical

Bayesian framework, which has been extensively utilized in the disease surveillance domain [20-22].

We attempt to estimate the relative risks of disease occurrence among geographical units $\left(\theta_{i t}\right)$ by means of statistical assessment. Formally,

$$
\begin{aligned}
& \text { Data } \quad: \quad Y_{i t} \mid \lambda_{i t} \sim \operatorname{Poisson}\left(\lambda_{i t}\right), \quad \lambda_{i t}=E_{i t} \times \theta_{i t} \\
& E_{i t}=R_{t}^{(\text {standardized })} P_{i t} \\
& R_{t}^{(\text {standardized })}=\frac{\sum_{i} Y_{i t}}{\sum_{i} P_{i t}}=\frac{\text { total conf irmed cases at time } t}{\text { sum of population of each district at time } t} \\
& \text { Process : } \quad \theta_{i t} \mid \psi \sim \pi(\cdot \mid \psi), \quad \eta_{i t}=\log \left(\theta_{i t}\right) \\
& \eta_{i t}=\beta_{0}+\sum_{j=1}^{p} \beta_{j} X_{i, j, t}+s_{i}+\gamma_{\text {week }(t)}+\gamma_{\operatorname{month}(t)}+\delta_{i t} \\
& s_{i} \sim B Y M 2 \text { specification }\left(\phi, \tau_{s}^{-1}\right) \\
& \gamma_{\text {week }(t)} \mid \gamma_{\text {week }(t-1)} \sim \operatorname{Normal}\left(\gamma_{\text {week }(t-1)}, \tau_{\text {week }}^{-1}\right) \\
& \gamma_{\text {month }(t)} \mid \gamma_{\text {month }(t-1)} \sim \operatorname{Normal}\left(\gamma_{\text {month }(t-1)}, \tau_{\text {month }}^{-1}\right) \\
& \delta_{i t} \sim \operatorname{Normal}\left(0, \tau_{\delta}^{-1}\right) \\
& \text { Parmeter: } \quad \psi \sim f(\cdot), \psi=\left(\beta_{0}, \beta_{j}, \tau_{\text {week }}, \tau_{\text {month }}, \tau_{\delta}, \tau_{s}, \phi\right)^{T} \\
& \beta_{0} \sim \operatorname{Normal}\left(0,10^{4}\right) \\
& \beta_{j} \sim \operatorname{Normal}\left(0,10^{4}\right) \text { for } j=1,2, \cdots, p \\
& \operatorname{Prob}\left(\left(1 / \sqrt{\tau_{\text {week }}}\right)>1\right)=0.01 \\
& \operatorname{Prob}\left(\left(1 / \sqrt{\tau_{\text {month }}}\right)>1\right)=0.01 \\
& \operatorname{Prob}\left(\left(1 / \sqrt{\tau_{\delta}}\right)>1\right)=0.01 \\
& \operatorname{Prob}\left(\left(1 / \sqrt{\tau_{s}}\right)>(0.5 / 0.31)\right)=0.01 \\
& \operatorname{Prob}(\phi<0.5)=2 / 3
\end{aligned}
$$


where the observed COVID-19 case, $Y_{i t}$, for each municipality, $i$, on a week, $t$, is assumed to be following a Poisson distribution. The Poisson parameter, $\lambda_{i t}$, is further decomposed by the product of the expected value (or an offset), $E_{i t}$ and the relative risk, $\theta_{i t}$. Here, we use indirect standardization of $E_{i t}$ to adjust for differences in exposure [20, 22, 24]. As shown in Equation (1), $E_{i t}$ is composed of $R_{t}^{(\text {standardized) }}$, the rate in the standard population (total number of cases divided by total population in all areas at time $t$ ) and $P_{i t}$, the population of area $i$ at time $t$. The overall disease rate at time $t$, $R_{t}^{(\text {standardized })}$, becomes a baseline that proportionately allocates the disease risk with respect to local population. $\eta_{i t}$ denotes a linear predictor, consisting of the fixed effects characterized by an intercept $\left(\beta_{0}\right)$ and the inner product between covariates $\left(X_{i, \mathrm{j}, t}\right)$ and coefficients $\left(\beta_{\mathrm{j}}\right)$, and of the random (latent) effects originating from spatial dependency $\left(s_{i}\right)$, non-linear weekly temporal trends $\left(\gamma_{w e e k(t)}\right)$, nonlinear monthly temporal influence $\left(\gamma_{\text {month }(t)}\right)$, and spatiotemporal interaction $\left(\delta_{i t}\right)$. Each $\tau$ stands for a precision parameter, an inverse of the variance. $\phi$ is a mixing parameter of the BYM2 model that is related to the marginal contribution of the scaled spatially structured random component [23].

As described, the relative risks are systemically broken down into several components such as fixed and random effects. The random components are further decomposed into spatially correlated, temporally structured, and unstructured spatiotemporal effects. To consider spatial correlation, we employ a modified Besag-York-Mollié model (BYM2, a reparameterization of the BYM model) and the weakly informative penalized complexity (PC) prior [23-24]. For mathematical details, one may refer to Riebler et al. [25], Simpson et al. [23], and Fuglstad et al. [26]. Regarding temporal influences, we adopt non-linear dynamic trends characterized by the random walk of order one (RW1) process on both weekly and monthly shifts [27]. Finally, we assume unstructured spatiotemporal interaction (Knorr-Held type I specification) [28] that captures unexplained variability in residuals after accounting for both fixed and random effects [22, 27].

In defining PC priors for each random effect, we adopt the probability statement $\operatorname{Prob}((1 / \sqrt{\tau})>L)=a$ and $\operatorname{Prob}(\phi<U)=b$, where $L$ and $U$ are reasonable thresholds for parameters considered, while $a$ and $b$ are their corresponding probabilities. Based on this 
understanding, we present Equation (3) which provides the detailed application of PC priors to the marginal precision parameters $\left(\tau_{\text {week }}, \tau_{\text {month }}, \tau_{s}, \tau_{\delta}\right)$ and the mixing parameter $(\phi)$.

\section{RESULTS}

\section{Model outcomes}

Prior to delving into detailed results, it is worth noting that our formal assessment is based on the weekly aggregates of COVID-19 cases of each municipality from January 24 to December 31, 2020 (i.e., 77 municipalities $\times 50$ weeks $=3,850$ observations). To account for differences in exposure of COVID-19 infection, we rely upon the expected value as given in Equation (1). We employ RINLA, an efficient alternative to the traditional Markov chain Monte Carlo (MCMC) simulation for Bayesian models, to implement a series of Bayesian spatiotemporal models [29].

Table 1 shows the results of our optimal model based on the goodness of fit measures such as the deviance information criterion (DIC) and the widely applicable information criterion (WAIC) [3031]. The size of individual municipalities and population density were considered as fixed effects in this study. The size of cities is estimated to have no important association with COVID-19 risks as the large variability of $95 \%$ credible interval indicates. Conversely, the influence of population density appears to be positive, implying an elevated relative risk of infectious diseases.

\section{[Table 1. Here]}

Meanwhile, we present the posterior distribution of different types of unobserved heterogeneity in explaining the random fluctuation of the relative risks of COVID-19. The posterior mean of the mixing parameter $(\phi)$ is estimated to be 0.117 , implying that the marginal contribution of the scaled spatially structured random component is small that reveals less spatial dependency of the risks (i.e., $\sqrt{0.117}=0.342$, the relative contribution of the scaled spatially structured random effect is $34.2 \%$ of overall spatial influences, $\left.s_{i}\right)$. Regarding temporal influences, we considered two random walks of order one (RW1) priors for weekly and monthly effects. The higher precision of their respective hyperparameters $\left(\tau_{\text {week }}, \tau_{\text {month }}\right)$ suggests that the temporal effects are relatively small. This is also depicted in Figure 2 which shows the posterior distribution of the standard deviation of 
the latent effects. It can be achieved by transforming each precision parameter to one with the standard deviation. In doing so, we employ the R-INLA function inla.tmarginal() to facilitate the conversion of parameters [24]. Moreover, the random fluctuation by spatiotemporal interaction is identified, whose contribution to overall variability of the relative risks turns out to be the largest.

\section{[Figure 2. Here]}

From a different perspective, we provide the marginal posterior mean and a 95\% credible interval of the temporal trend as well as the spatiotemporal interaction in Figure 3. The left panel of Figure 3 (A) shows that the weekly time trend is not linear, supporting a nonparametric pattern (i.e., justifying our adoption of the RW1 process for temporally structured random effects). The marginal contribution of the temporal effect on the relative risks is small, as the magnitude (value on the y-axis) indicates. In contrast, the influence of spatiotemporal interaction on the response seems to be high (right panel of Figure 3 (B)), capturing the unexplained residual variability of the COVID-19 relative risks for municipalities over the study period.

\section{[Figure 3. Here]}

\section{Spatiotemporal evolution of relative risks}

Considering these findings, we present Figure 4 which shows the spatiotemporal evolution of the relative risks across municipalities over weeks. Six temporal points are chosen considering the representativeness that reflects seasonal variations and newly emerged clusters of COVID-19 infections. We selected Week4 as it was the first week that we confirmed positive COVID-19 cases in the GSA (Winter) while Week11 and Week23 (Spring) were included following the implementation of SD and enhanced SD policies. A surge of infectious cases was observed in Week34 (Summer). We observed stabilizing trends in Week44 (Fall) after the second wave. Finally, we witnessed a rebound attributed to winter cold, lowering their guard against COVID-19 infection, lack of awareness, and frequent smaller gatherings in Week52 (Winter).

\section{[Figure 4. Here]}


Regarding the interpretation of the results, it is worth noting that when a municipality has a relative risk greater (smaller) than 1, the city is regarded as an elevated risk (lower risk) region. The pattern of the evolution of the relative risks for each district shows that it is spatially and temporally structured rather than random. For example, the estimated posterior mean suggests that municipalities in the northern and southern parts of Gyeonggi province are likely to be relatively safer than those in Seoul at Week4. In Week11 and Week23, the patterns of boundary municipalities of Gyeonggi province seemed to continue, while we observed newly emerged areas of higher risks in western Seoul (central regions of the GSA). When it comes to Week34 and Week44, several districts of elevated COVID-19 risks emerged in the northern Gyeonggi province and in the southern parts of Seoul. The disease map exhibits a different spatial distribution at Week52, reflecting the evolution of risks over space and time. In that regard, we verified that districts with similar relative risks tend to be located together, implying some spatial dependency. The spatial pattern also changes with respect to temporal shifts, which further reveals a temporal correlation. This suggests that accounting for random effects in the modeling process gives rise to an improved understanding of the relative risk of local-level COVID-19 transmission across the GSA.

Meanwhile, we selected five districts (two in Seoul and three in Gyeonggi province) whose estimates of relative risks tended to be higher than other regions, to show their individual evolution over time. The estimated posterior mean of the relative risks was obtained for 50 weeks per municipality (i.e., 50 fitted values each). For each district, we applied a locally estimated scatterplot smoothing (LOESS) regression to obtain a smoother curve (Figure 5), which helped us better appreciate the evolution of the risks. As shown in Figure 5, none of the districts have similar patterns in general, but reveal individual specific evolution of the relative risks over time, reflecting the heterogeneous features of COVID-19 transmission. For example, a surge in Sujeong-gu occurred between Week10 and Week15, which is associated with religious gatherings, while the curve stabilized after this. Virus-prone facilities, such as gyms and saunas, are responsible for the surge in Seocho-gu, whereas a spike in Songpa-gu at Week53 originates from overcrowded correctional facilities, where highly transmissible viral infections can rapidly disseminate. 


\section{[Figure 5. Here]}

\section{DISCUSSIONS}

This research attempts to explore the local level spatiotemporal dynamics of COVID-19 spread within the capital regions of South Korea throughout the year 2020. For weekly accumulated cases per individual municipality over the study period ( 50 weeks in total), we consider the relative risks of COVID-19 transmission as a point of interest and attempt to account for uncertainties associated with them. To this end, we exploit a generalized linear mixed modeling method under a spatiotemporal hierarchical Bayesian framework, which is flexible enough to accommodate latent influences from the neighboring structure of administrative units, their serial correlation, and spatiotemporal interaction on top of fixed effects by covariates. We verify that the size of municipalities seems to have no importance on COVID-19 infectious risks, whereas more densely populated districts are likely to have an elevated risk. Regarding latent/random effects, our study reveals that spatiotemporal interaction dominates the overall variability of random influences, followed by spatial correlation, while the temporal correlation appears to be small. Based on these findings, we present the dynamic evolution of COVID-19 across the GSA, which helps us clarify unique patterns of the disease as well as regions of elevated risks for further intervention.

In this study, however, there are several limitations that should be acknowledged. We present different policy (counter)measures against COVID-19 in Figure 1. Frequent changes as well as varied policy measures make variable construction rather challenging. Thus, while in a formal modelling process, we do not account for their potential impacts on mitigating the spread. There might be possible lagged influences of policy measures on the infectious disease which can be further examined in a future research work. In addition, numerous studies have investigated the association between environments (e.g., air pollution and climates), built environments as well as sociodemographics, and COVID-19 transmission [32-35]. Unlike those previous efforts whose focus are on direct effects of various influential factors, our study rather concentrates upon indirect/latent influences of space and time to explain the spatiotemporal dynamics of COVID-19 infections. To justify our approach, we base our study design upon Cressie [36], who suggests that spatial random 
effects can explain missing covariates and unobserved heterogeneity. Put differently, the adoption of our spatiotemporal random effect model can account for influences from missing covariates such as policy measures, environments, and sociodemographic features in the residual term.

Nonetheless, we argue that our attempt can contribute to enhancing the understanding of local level transmission mechanisms of COVID-19 in densely populated regions. Using the GSA in South Korea as an example, our empirical study sheds light on the patterns of evolution of COVID-19 over time from a different perspective, complementing other works and leading to supporting evidencebased policy decisions against infectious diseases (e.g., developing customized preventive policy measures considering spatial disparities in infection risks). 


\section{References}

1. Who coronavirus (COVID-19) dashboard [Internet]. World Health Organization. World Health Organization; [cited 2021 Jun 10]. Available from: https://covid19.who.int/

2. Ministry of Health and Welfare Disease 19(COVID-19). Coronavirus (COVID-19), Republic of Korea(COVID-19) [Internet]. Coronavirus disease 19(COVID-19). [cited 2021 Jun 10]. Available from: http://ncov.mohw.go.kr/en/tcmBoardView.do?brdId=12\&amp;brdGubun=125\&amp;dataGub un=\&amp;ncvContSeq=4552\&amp;contSeq=4552\&amp;board_id=\&amp;gubun=

3. Coronavirus [Internet]. World Health Organization. World Health Organization; [cited 2021 Mar 1]. Available from: https://www.who.int/health-topics/coronavirus\#tab=tab_1

4. Lai C-C, Shih T-P, Ko W-C, Tang H-J, Hsueh P-R. Severe acute respiratory syndrome coronavirus 2 (SARS-COV-2) and coronavirus disease-2019 (COVID-19): The epidemic and the challenges. International Journal of Antimicrobial Agents. 2020;55(3):105924.

5. OECD Regional Outlook 2021: Addressing COVID-19 and moving to net zero greenhouse gas emissions. Paris: OECD Publishing; 2021.

6. Briz-Redón Á, Serrano-Aroca Á. A spatio-temporal analysis for exploring the effect of temperature on COVID-19 early evolution in Spain. Science of The Total Environment. 2020;728:138811.

7. Briz-Redón Á. The impact of modelling choices on modelling outcomes: A spatio-temporal study of the association between covid-19 spread and environmental conditions in Catalonia (Spain). Stochastic Environmental Research and Risk Assessment. 2021;

8. Kang D, Choi H, Kim J-H, Choi J. Spatial epidemic dynamics of the covid-19 outbreak in China. International Journal of Infectious Diseases. 2020;94:96-102.

9. Valente F, Laurini MP. Estimating spatiotemporal patterns of deaths by covid-19 outbreak on a global scale. BMJ Open. 2021;11(8).

10. Kim S, Castro MC. Spatiotemporal pattern of COVID-19 and government response in South Korea (as of May 31, 2020). International Journal of Infectious Diseases. 2020;98:328-33.

11. Lee JH, Park MS, Lee SW, Spatiotemporal Evolution and the Diffusion Pattern of COVID19 in South Korea in Three Waves. The Korea Spatial Planning Review 2021;110:43-62 (Korean)

12. Shim E, Tariq A, Chowell G. Spatial variability in reproduction number and doubling time across two waves of the COVID-19 pandemic in South Korea, February to July, 2020. International Journal of Infectious Diseases. 2021;102:1-9.

13. Lee W, Hwang S-S, Song I, Park C, Kim H, Song I-K, et al. Covid-19 in South Korea: Epidemiological and spatiotemporal patterns of the spread and the role of aggressive diagnostic tests in the early phase. International Journal of Epidemiology. 2020;49(4):110616.

14. Lee T, Kwon H-D, Lee J. The effect of control measures on covid-19 transmission in South Korea. PLOS ONE. 2021;16(3).

15. Ministry of Health and Welfare Disease 19(COVID-19). Coronavirus (COVID-19), Republic of Korea(COVID-19) [Internet]. Coronavirus disease 19(COVID-19). [cited 2021 Apr 1]. Available from: http://ncov.mohw.go.kr/en/tcmBoardView.do?brdId=12\&amp;brdGubun=125\&amp;dataGub un=\&amp;ncvContSeq=2996\&amp;contSeq=2996\&amp;board_id=\&amp;gubun=\#

16. Park I-N, Yum HK. Stepwise strategy of social distancing in Korea. Journal of Korean Medical Science. 2020;35(28). 
17. All about Korea's response to COVID-19 [Internet]. Ministry of Foreign Affairs, Republic of Korea. [cited 2021 Apr 1]. Available from:

https://www.mofa.go.kr/viewer/skin/doc.html?fn=20201021031300238.pdf\&rs=\%2Fviewer $\% 2$ Fresult $\% 2 \mathrm{~F} 202103$

18. New social distancing system will have 5 tiers [Internet]. Korea joongAng Daily. [cited 2021 Feb 1]. Available from:

https://koreajoongangdaily.joins.com/2020/11/01/national/socialAffairs/virus-covid-socialdistancing/20201101191400439.html

19. National Spatial Data Infrastructure Portal (NSDI) [Internet]. nsdi.go.kr. [cited 2021 Feb 1]. Available from: http://www.nsdi.go.kr/lxportal/?menuno=3085

20. Lawson A. Bayesian disease mapping: Hierarchical modeling in spatial epidemiology. Boca Raton: Chapman \& Hall/CRC; 2021.

21. Congdon P. Bayesian hierarchical models: With applications using R. Boca Raton: Chapman \& Hall/CRC; 2021.

22. Martinez-Beneito, M. A., Botella-Rocamora, P. Disease mapping: From foundations to Multidimensional Modeling. APPLE ACADEMIC Press INC; 2019.

23. Simpson D, Rue H, Riebler A, Martins TG, Sørbye SH. Penalising model component complexity: A principled, practical approach to constructing priors. Statistical Science. 2017;32(1).

24. Moraga P. Geospatial Health Data: Modeling and visualization with R-INLA and shiny. Boca Raton, FL: CRC Press, Taylor \& Francis Group; 2020.

25. Riebler A, Sørbye SH, Simpson D, Rue H. An intuitive bayesian spatial model for disease mapping that accounts for scaling. Statistical Methods in Medical Research.

2016;25(4):1145-65.

26. Fuglstad G-A, Simpson D, Lindgren F, Rue H. Constructing priors that penalize the complexity of Gaussian Random Fields. Journal of the American Statistical Association. 2018;114(525):445-52.

27. Blangiardo M, Cameletti M. Spatial and Spatio-Temporal Bayesian models with R-INLA. Chichester: Wiley; 2015.

28. Knorr-Held L. Bayesian modelling of inseparable space-time variation in disease risk. Statistics in Medicine. 2000;19(17-18):2555-67.

29. Rue H, Martino S, Chopin N. Approximate bayesian inference for latent gaussian models by using integrated nested laplace approximations. Journal of the Royal Statistical Society: Series B (Statistical Methodology). 2009;71(2):319-92.

30. Spiegelhalter DJ, Best NG, Carlin BP, van der Linde A. Bayesian measures of model complexity and fit. Journal of the Royal Statistical Society: Series B (Statistical Methodology). 2002;64(4):583-639.

31. Wang, X., Yue, R. Y., Faraway, J. J. Bayesian regression modeling with INLA. S.1.: CRC PRESS; 2020.

32. Anand U, Cabreros C, Mal J, Ballesteros F, Sillanpää M, Tripathi V, et al. Novel coronavirus disease 2019 (covid-19) pandemic: From transmission to control with an interdisciplinary vision. Environmental Research. 2021;197:111126.

33. Marquès M, Domingo JL. Positive association between outdoor air pollution and the incidence and severity of COVID-19. A review of the recent scientific evidences. Environmental Research. 2022;203:111930. 
34. Raymundo CE, Oliveira MC, Eleuterio Tde, André SR, da Silva MG, Queiroz ER, et al. Spatial analysis of covid-19 incidence and the sociodemographic context in Brazil. PLOS ONE. 2021;16(3).

35. Lym Y, Kim K-J. Exploring the effects of PM2.5 and temperature on COVID-19 transmission in Seoul, South Korea. Environmental Research. 2022;203:111810.

36. Cressie NA. Statistics for Spatial Data. Hoboken, NJ: John Wiley \&amp; Sons, Inc; 2015. 
Table 1. Regression outcomes

\begin{tabular}{|c|c|c|c|c|c|c|}
\hline & \multicolumn{6}{|c|}{ Dependent variable: weekly aggregates of confirmed cases } \\
\hline & Mean & S.D & $2.5 \%$ & $50 \%$ & $97.5 \%$ & Mode \\
\hline \multicolumn{7}{|l|}{ Fixed effects } \\
\hline Intercept & -1.931 & 0.338 & -2.601 & -1.93 & -1.268 & -1.928 \\
\hline Log (Population density) & 0.178 & 0.045 & 0.089 & 0.179 & 0.268 & 0.179 \\
\hline CitySizeLevel2 ${ }^{1)}$ & 0.12 & 0.228 & -0.326 & 0.119 & 0.57 & 0.117 \\
\hline CitySizeLevel3 $^{1)}$ & -0.036 & 0.235 & -0.498 & -0.037 & 0.427 & -0.038 \\
\hline 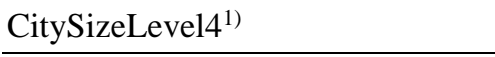 & 0.068 & 0.254 & -0.424 & 0.066 & 0.575 & 0.061 \\
\hline \multicolumn{7}{|l|}{ Random effects (Hyperparameters) } \\
\hline Precision of BYM22) & 5.458 & 1.124 & 3.42 & 5.418 & 7.79 & 5.373 \\
\hline Mixing parameter of $\mathrm{BYM} 2^{2)}$ & 0.117 & 0.094 & 0.01 & 0.092 & 0.36 & 0.029 \\
\hline Precision of Weekly effect ${ }^{3)}$ & 476.949 & 892.856 & 30.49 & 230.35 & 2474.95 & 75.895 \\
\hline Precision of Monthly effect ${ }^{3)}$ & 301.298 & 420.782 & 21.04 & 174.942 & 1350.95 & 55.632 \\
\hline Precision of Spacetime interaction & 1.969 & 0.094 & 1.78 & 1.973 & 2.14 & 1.992 \\
\hline \multicolumn{7}{|l|}{ Goodness of fit } \\
\hline DIC & 13986.72 & & & & & \\
\hline WAIC & 13780.51 & & & 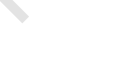 & & \\
\hline Marginal log-Likelihood & -8121.01 & & & & & \\
\hline
\end{tabular}

Note: 1) Reference category: CitySizeLevel1. In defining the size of each municipality, we considered the magnitude of the population (i.e., Level1 $(<100 \mathrm{~K})$, Level2 $(<250 \mathrm{~K})$, Level3 $(<500 \mathrm{~K})$, and Level4 $(500 \mathrm{~K}+))$.

2) There are two parameters (marginal precision and mixing parameter) in the BYM2 model.

3) The inverse of variance is a precision (i.e., $\tau^{-1}=\sigma^{2}$ ). We consider both weekly and monthly structured temporal influences.

4) Number of observations $(\mathrm{N} \times \mathrm{T}): 77$ districts $\times 50$ weeks $=3850$. 


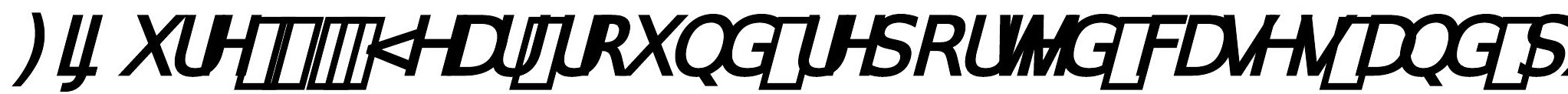

Type of COVID-19 virus

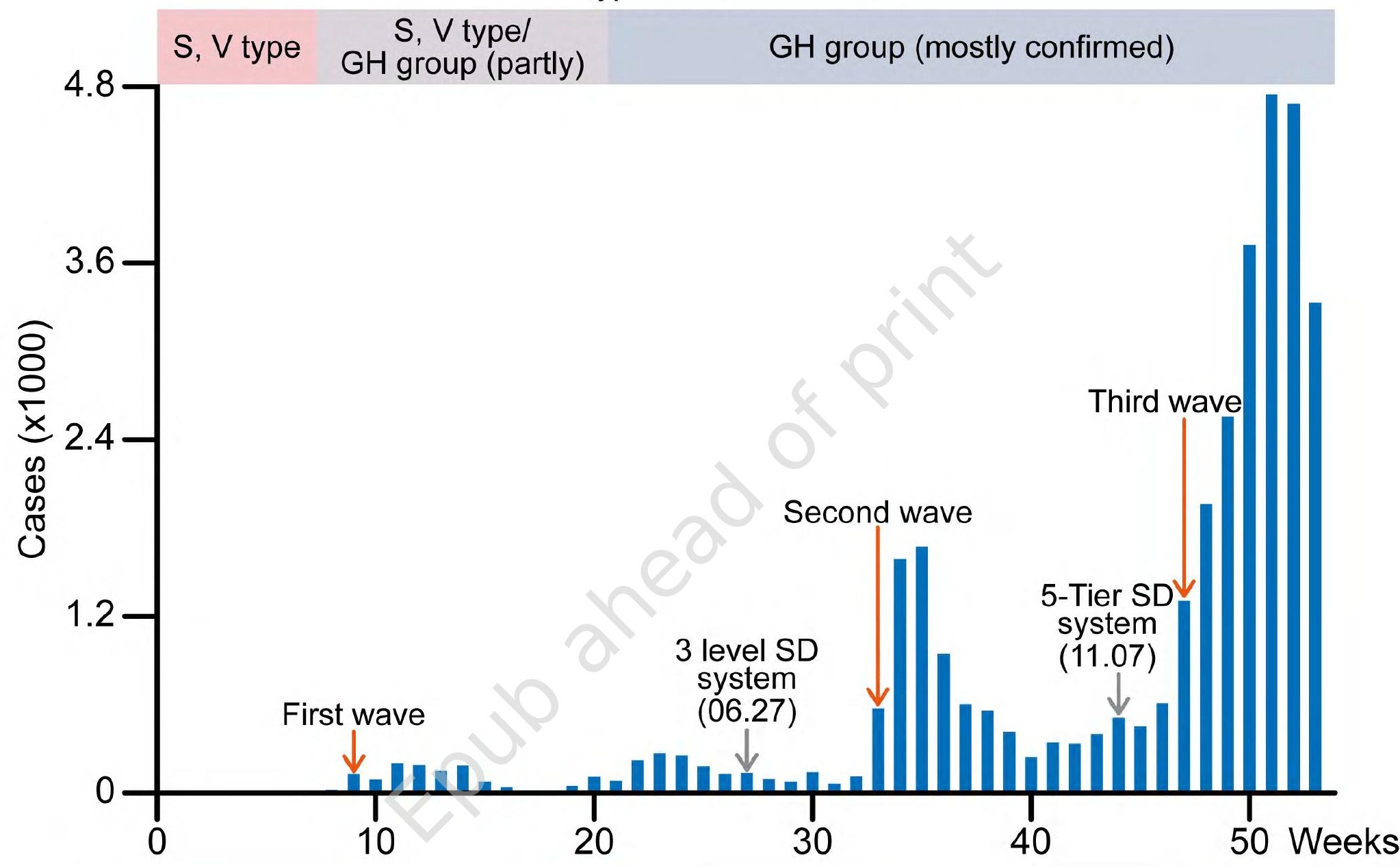

SD policy

SD Enhanced SD

Distancing to daily life

Level 1 Level 1.5 Level 2 Enhanced level 2 Level 2.5 


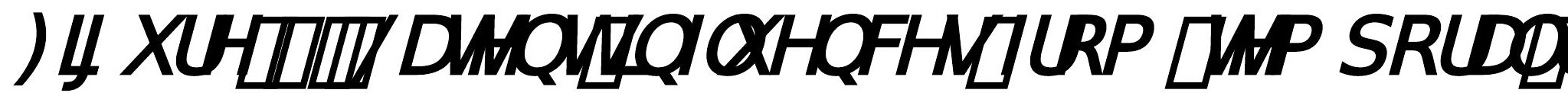
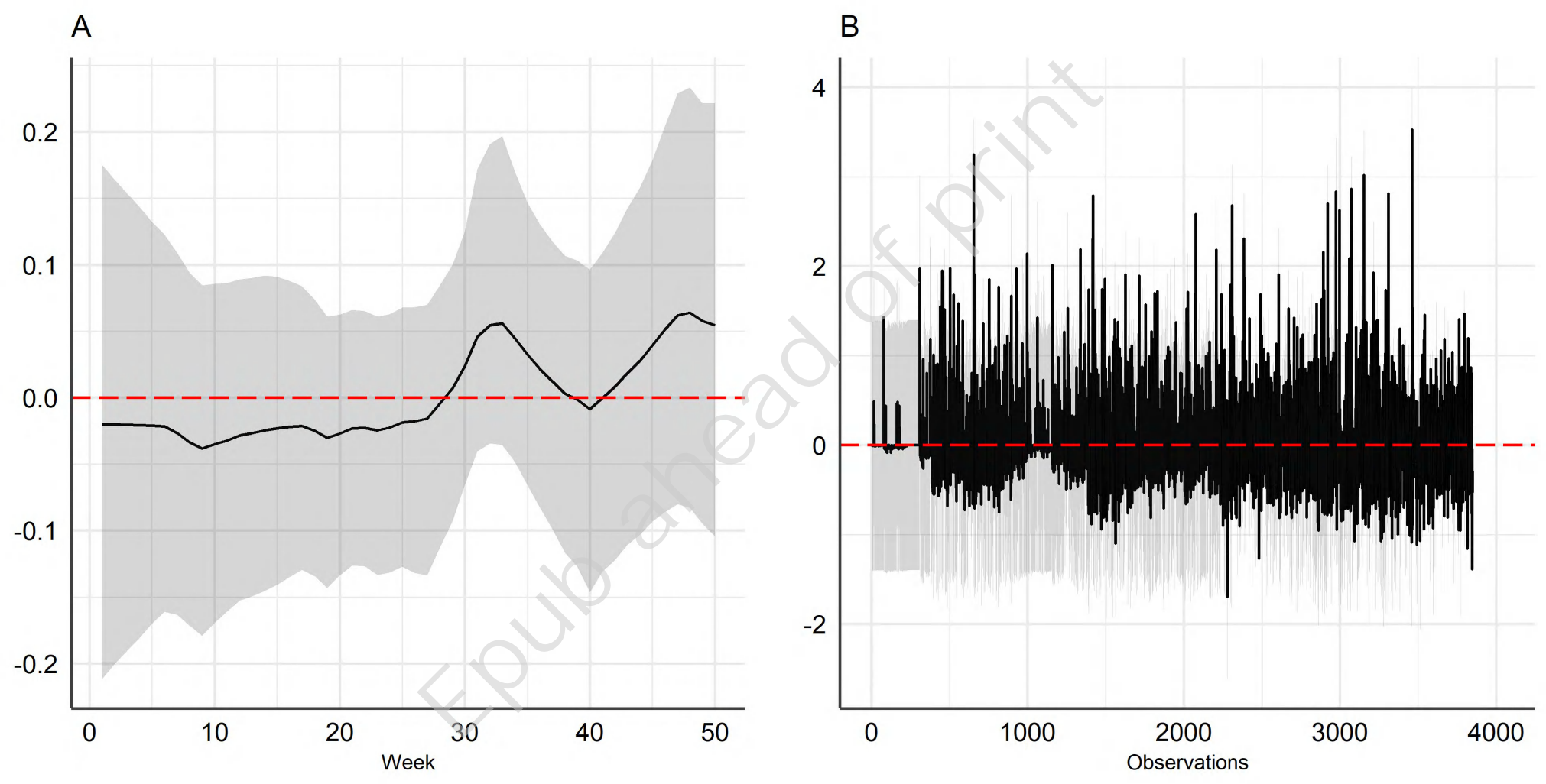


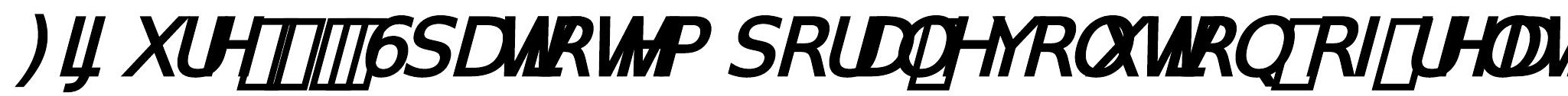
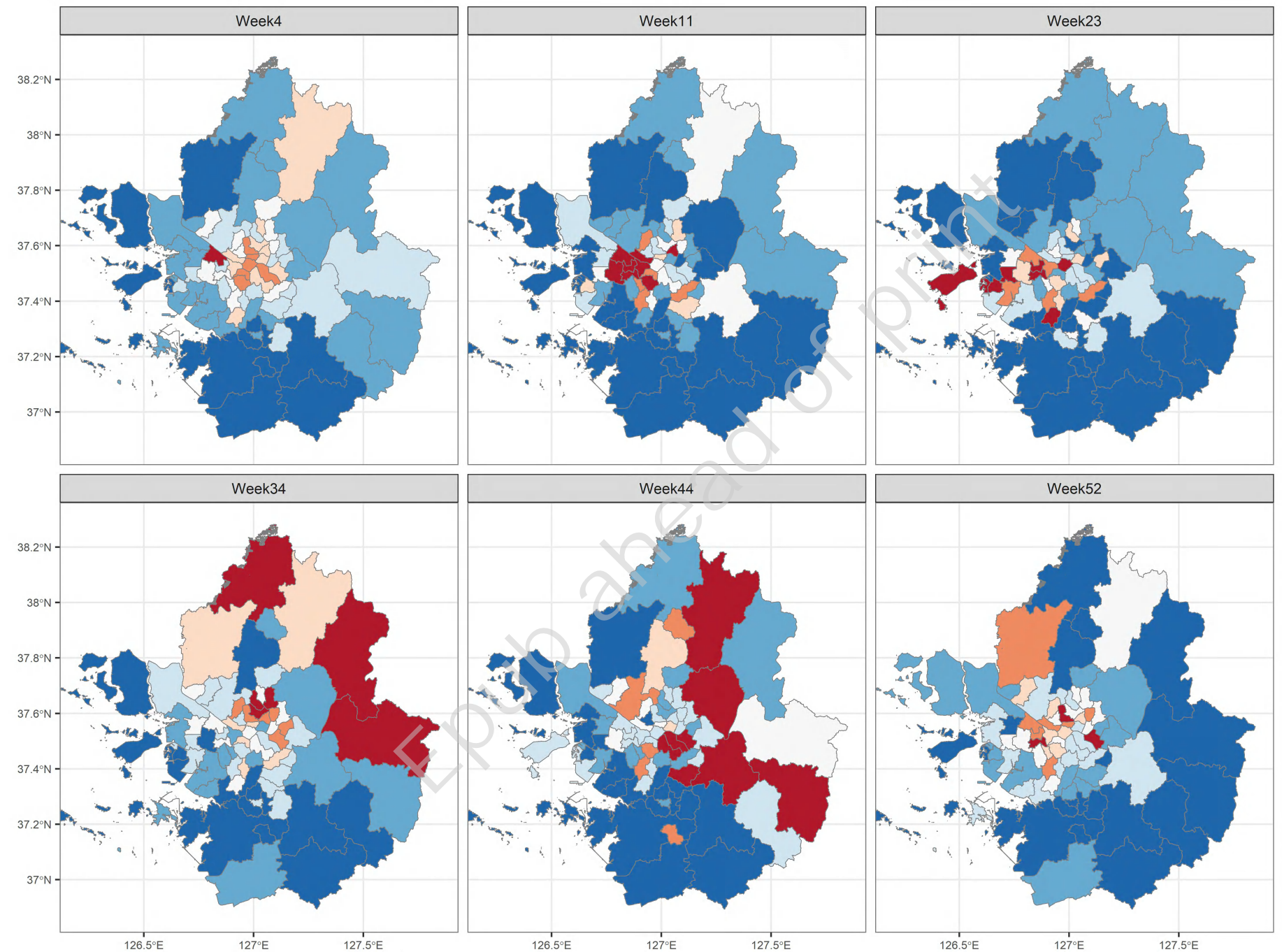

Relative

Risks

$\square>2$

$(1.5,2]$
$-(1.25,1.5]$

$(1.25,1.5]$
$(1,1.25]$

$(1,1.25]$
$(0.75,1]$

$(0.5,0.75$

$<0.5$ 
